\title{
The Prison and Transition Health (PATH) Cohort Study: Study Protocol and Baseline Characteristics of a Cohort of Men with a History of Injecting Drug Use Leaving Prison in Australia
}

\author{
Amy Kirwan • Michael Curtis • Paul Dietze • Campbell Aitken • Emma Woods • \\ Shelley Walker • Stuart Kinner • James Ogloff • Tony Butler • Mark Stoové (iD
}

Published online: 15 April 2019

(C) The Author(s) 2019

\begin{abstract}
People who inject drugs (PWID) are disproportionately represented among individuals who experience imprisonment and often have more complex physical and mental health needs than people in prison without injecting histories. The trajectories of PWID after prison release are poorly understood, hampering the development of effective strategies to address their distinct health needs. The Prison and Transition Health (PATH) Cohort Study is characterising the post-release trajectories of incarcerated male PWID in Victoria, Australia. We outline study methodology and baseline characteristics of participants prior to their release. Four hundred participants were recruited from three prisons and completed researcher-administered baseline
\end{abstract}

A. Kirwan · M. Curtis · P. Dietze · C. Aitken · E. Woods · S. Walker · M. Stoové $(\square)$

Behaviours and Health Risks Program, Public Health Discipline, Burnet Institute, 85 Commercial Rd, Melbourne, VIC 3004, Australia

e-mail: mark.stoove@burnet.edu.au

M. Curtis · P. Dietze · C. Aitken · M. Stoové

School of Public Health and Preventive Medicine, Monash

University, Melbourne, Australia

S. Walker · S. Kinner

National Drug Research Institute, Curtin University, Perth, Western Australia

S. Kinner

Centre for Adolescent Health, Murdoch Children's Research Institute, Melbourne, Australia interviews covering socio-demographics, social supports, physical health, mental health, alcohol and other drug use, and pre-release and transitional service utilisation. The median age among participants was 36 years (IQR 30-42), and they reported a median of five (IQR 3-9) previous adult incarcerations. Almost half $(49 \%)$ were reliant on government payments prior to incarceration. One quarter $(25 \%)$ of participants reported removal from their parents' care as children and $64 \%$ reported being a parent or primary caregiver to children. Most participants $(81 \%)$ reported a previous mental health diagnosis and $44 \%$ reported three or more diagnoses. The most common drugs injected prior to incarceration were crystal methamphetamine (80\%) and

\author{
S. Kinner \\ Centre for Mental Health, Melbourne School of Population and \\ Global Health, University of Melbourne, Melbourne, Australia \\ S. Kinner \\ Griffith Criminology Institute, Griffith University, Brisbane, \\ Australia \\ S. Kinner \\ Mater Research Institute-UQ, University of Queensland, Mount \\ Gravatt, Australia \\ J. Ogloff \\ Centre for Forensic Behavioural Science, Swinburne University of \\ Technology and Forensicare, Melbourne, Australia \\ T. Butler \\ Kirby Institute, University of New South Wales, Sydney, Australia
}


heroin $(62 \%)$, and most $(85 \%)$ reported being under the influence of drugs at the time of committing offences for which they were currently incarcerated. Injecting drug use during their current sentence was reported by $40 \%$ of participants, and $48 \%$ reported engaging with some form of drug treatment during their current sentence. Study participants are characterised by significant mental health and substance use morbidities, social disadvantage and criminogenic histories that present challenges for the provision of post-release support services. Data from the PATH Cohort Study will help inform strategies to improve the health and social outcomes of this population.

Keywords Prison · Injecting drug use $\cdot$ Cohort study

\section{Background}

People who inject drugs (PWID) are disproportionately represented among those who experience imprisonment [1]. Among people incarcerated in Australia, approximately half report ever injecting drugs [2] and approximately one quarter report injecting in the month prior to prison reception [3, 4]. Prisoners have considerably more complex physical and mental health needs $[2,3$, $5,6]$ and experience greater economic, psycho-social and educational disadvantage than the general population [7]. Those with a history of injecting drug use (IDU) have increased risk of a range of additional adverse health outcomes, including blood-borne virus (BBV) infections $[2,4,8]$ and overdose [9, 10]. In Australia, these overlapping risks disproportionately affect $\mathrm{Ab}$ original and/or Torres Strait Islanders, who are overrepresented in prison populations [11, 12], more likely to use injectable illicit drugs [13], and also experience greater socio-economic disadvantage and earlier contact with the criminal justice system [14].

Challenges associated with transitioning from prison to the community, including financial and relationship stress, social isolation and stigma, can be heightened for people with histories of drug dependence and mental and physical health comorbidities [15-17] and contribute to post-release service access barriers [18]. For those with drug dependence histories, return to drug use [19] and recidivism [20] following release and reincarceration are also common. Approximately $40 \%$ of all people released from prison in Australia return within 2 years [21], with 2-year reincarceration rates among those with IDU histories estimated to be as high as $84 \%$ [22].

Extensive literature describes the health and social challenges of people released from custody; however, the trajectories of people released from prison who were engaging in regular IDU in the months immediately prior to incarceration remain poorly understood. Given their health and social vulnerabilities and high rates of recidivism, the scarcity of studies describing the postrelease natural history of this group represents a significant gap. To date, the few cohort studies of people released from prison in Australia have provided limited insights given they recruited general (i.e. unselected) prisoner populations [23, 24], relied exclusively on record linkage $[22,25,26]$, recruited small samples with short follow-up $[15,20]$, or recruited in the community in the weeks after release [27]. These studies also lacked the depth of information needed to detail the temporal relationships between incarceration, IDU, physical and mental health, health service utilisation and recidivism.

The Prison and Transition Health (PATH) Cohort Study aims to characterise the prison-to-community trajectories of incarcerated males in Victoria, Australia, who report regular IDU in the months immediately prior to incarceration. This paper outlines the PATH Cohort Study research protocol and describes the baseline (prerelease) characteristics of the sample.

\section{Methods}

\section{Study Design}

This prospective cohort study recruited incarcerated males nearing the end of their sentences who had a self-reported history of regular IDU in the months immediately prior to incarceration. Data collection consisted of in-depth quantitative interviews, blood specimen collection and blood-borne virus testing, and record linkage to health, housing and justice databases. Primary data collection for the study included extensive quantitative interviews conducted at baseline (prerelease) and 3, 12 and 24 months after release, which constitute the primary study data. Blood specimen collection occurred at baseline, 12 months and 24 months. Data linkage is planned at 2, 5 and 10 years post-release to describe long-term service interactions and health, social and criminal justice outcomes. This paper 
Table 1 Summary of variables collected at baseline interview

\begin{tabular}{|c|c|}
\hline Domain & Content \\
\hline Socio-demographics & $\begin{array}{l}\text { Date of birth } \\
\text { Country of birth } \\
\text { Language spoken } \\
\text { Aboriginal and Torres Strait } \\
\quad \text { Islander status } \\
\text { Sexual identity } \\
\text { Relationship status } \\
\text { Accommodation status } \\
\text { Children } \\
\text { Level and type of education } \\
\text { completed } \\
\text { Income prior to prison } \\
\text { Employment prior to prison }\end{array}$ \\
\hline Social support & $\begin{array}{l}\text { Recent contact with family and } \\
\text { friends } \\
\text { Availability of social supports } \\
\text { Family history }\end{array}$ \\
\hline Physical health & $\begin{array}{l}\text { Current health status } \\
\text { Physical illnesses/conditions } \\
\text { Viral infections (status, testing } \\
\text { and treatment) } \\
\text { Medications } \\
\text { Disabilities and injuries }\end{array}$ \\
\hline Mental health & $\begin{array}{l}\text { Current mental health status } \\
\text { (GHQ-12) [46] } \\
\text { Mental health service usage } \\
\text { Diagnoses } \\
\text { Suicide and self-harm }\end{array}$ \\
\hline $\begin{array}{l}\text { Alcohol and other drugs and } \\
\text { related behaviours }\end{array}$ & $\begin{array}{l}\text { Alcohol use prior to prison } \\
\text { (AUDIT C) [47] } \\
\text { Licit and illicit drug use prior to } \\
\text { and in prison } \\
\text { Injecting drug use (history, risks, } \\
\text { behaviours) } \\
\text { Use of OST } \\
\text { Use of other AOD services and } \\
\text { programs } \\
\text { Overdose } \\
\text { Level of Service } \\
\text { Inventory-Revised: Screening } \\
\text { Version (LSI-R:SV) [48] }\end{array}$ \\
\hline $\begin{array}{l}\text { Pre-release and transitional } \\
\text { services and arrangement }\end{array}$ & $\begin{array}{l}\text { Use of programs and services } \\
\text { Plans for release } \\
\text { Concerns about release } \\
\text { Expectations after release }\end{array}$ \\
\hline
\end{tabular}

describes the sample on the basis of their responses to baseline interviews which were conducted in prison.

\section{Setting}

Between September 2014 and June 2016, participants were recruited from three male prisons in the Australian state of Victoria. Operational limitations in the state's women's prisons at the time of data collection prevented planned recruitment of female participants. Recruitment prisons were selected to enhance sample heterogeneity by geography (metropolitan, regional) and security level (minimum, medium, maximum security). Recruitment targets at each prison aimed to reflect a proportional representation of the prison population size at each site. The male prisoner population in Victoria in 2016 was estimated at 6,644 [28] and the minimum, medium and maximum security recruitment prisons accommodated an estimated 242, 552 and 1067 prisoners, respectively [29].

\section{Eligibility Criteria}

Individuals were eligible to participate if they selfreported injecting drugs at least once a month in the 6 months before incarceration, were being aged 18 years or over, ${ }^{1}$ were a sentenced prisoner (i.e. not on remand), consented to participate in four face-to-face interviews (in-prison baseline and three follow-up interviews in the community), provide blood specimens, be regularly contacted by researchers over the follow-up period and record linkage for 10 years following recruitment.

Baseline interviews were scheduled for within 12 weeks of participants' expected release dates, which were determined using a combination of recorded endof-sentence dates and prisoner self-report of anticipated early release on parole, validated by prison staff at the time of recruitment. When release was delayed 12 weeks beyond baseline interviews, short catch-up interviews were conducted to update time-variant pre-release data.

\section{Recruitment and Consent}

Recruitment criteria of recent pre-incarceration IDU prevented random sampling using prison administrative records. To recruit participants, researchers engaged

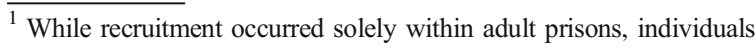
who are under 18 years are occasionally detained within these settings.
} 
directly with prisoners at medical centres during dosing times for opioid substitution therapy (OST), at alcohol and other drugs (AOD) therapeutic group sessions, through prison workplaces and in prison units/cell blocks. Researchers engaged peer representatives and staff involved in program delivery to prisoners with IDU histories to promote the study and presented at 'town hall meetings' (regular meetings of prisoners and staff), educational programs and prison workplaces. Study posters were displayed in general access areas of prisons. Those interested in participating who were not engaged directly by researchers completed 'expression of interest' forms, which they could submit directly to researchers, clinical or program staff, or place in secure mailboxes in prisons. These forms helped protect confidentiality and provided consent for researchers to contact prisoners and discuss the study in accordance with prison operations and ethics committee protocols. Researchers screened potential participants for eligibility, and once eligibility was established, a baseline interview was scheduled.

During the informed consent process, researchers verbally reinforced key elements of the written participant information and consent forms. The concept and process of record linkage, including participant confidentiality, and types of information sought from data custodians, was discussed with participants. In accordance with government requirements, participants completed a separate form to consent to data linkage to their federal medical records. Pre and post-test discussions occurred with all participants when baseline blood samples were collected, with test results delivery scheduled following release.

\section{Baseline Data Collection}

Researchers used electronic tablets to administer quantitative questionnaires, and data were downloaded into an electronic database (Mobile Data Studio Software) [30]. Questionnaires covered six domains: socio-demographics, social support, physical health, mental health, alcohol and other drug use and related behaviours, and pre-release and transitional services and arrangements. Items were adapted from those used in a cohort study of community-recruited PWID [31] and tested in a pilot study [20], alongside standardised and validated scales (Table 1). Mean duration of interviews was $45 \mathrm{~min}$ ( $\mathrm{SD}=12 \mathrm{~min}$, range 26-73 $\mathrm{min}$ ).
A dry blood spot finger-prick blood specimen was collected for hepatitis $\mathrm{C}$ antibody testing using commercially available assays. HIV testing was not performed due to the low prevalence of HIV among Victorian prison populations [4]. Hepatitis B testing was not performed due to budget limitations and the cost of dry blood spot assays.

Contact-tracing details were collected on paperbased forms and stored in an electronic database separate to survey data. To facilitate follow-up, researchers collected detailed participant identifying information (full name, date of birth, alias/street name, expected residential address, expected telephone numbers after release) and secondary contact details for friends, relatives, services or specific workers they anticipated contacting after release.

Participants were not reimbursed for baseline interviews in prison in accordance with the Corrections Victoria guidelines.

\section{Follow-up Data Collection}

Researchers are conducting follow-up interviews at 3, 12 and 24 months after release from prison (3- and 12month interviews complete at time of writing) and collecting venous blood samples for hepatitis B and hepatitis $\mathrm{C}$ antibody and virus testing (including RNA tests to determine chronic/active infections) at 12 and 24 months. Researchers provide blood test results to participants where possible and offer referral to hepatitis care and treatment providers.

Follow-up questionnaires are adapted from those administered at baseline, removing time-invariant items and adding or adapting items to capture prospectively occurring events and to reflect differences between the community and prison environments. Participants receive AUD40 as a cash payment for each follow-up interview completed in the community.

Researcher contact with participants during follow-up occurs via regular phone, email and social media contact, or via secondary contacts described above. Researchers also receive information on whether a participant who cannot be contacted has been reincarcerated during follow-up. Follow-up protocol permitted interviews to occur in any prison in Victoria in these circumstances. At the time of writing, all three waves of follow-up had greater than $50 \%$ participation and more than $80 \%$ of participants had completed at least one follow-up interview. 
Record Linkage

Record linkage will be conducted at 2, 5 and 10 years post-release across the following health and justice databases: Medicare and the Pharmaceutical Benefits Scheme (federally funded healthcare), state-wide mental health, alcohol and other drug treatment, ambulance, hospital emergency department, hospital admissions, housing services, police contact (arrest, charge, victim) and mortality. Participants consented to data linkage on prison program participation, including those related to addressing offending behaviour, drug use and use of prison health services, during the sentence in which they were recruited and future periods of incarceration over 10 years. Record linkage had not commenced at the time of writing.

\section{Ethics Approval}

The Victorian Department of Justice Human Research Ethics Committee and the Alfred Hospital Ethics Committee approved the study. Specific ethical and administrative approvals for record linkage were received from the Australian Government Department of Health, the Australian Institute of Health \& Welfare, Victoria Police, and the National Coronial Information System.

Analysis

In this paper, we present descriptive statistics to characterise the socio-demographics, physical and mental health, incarceration history, pre-incarceration offending, drug dependence treatment history and substance use of participants. These were generated using Stata SE Version 14.1 [32].

\section{Results}

Four hundred and nine participants were recruited over 21 months. Recruitment proved challenging within the prison setting due to various issues, including negotiating access to locations where researchers could engage potential participants directly, building trust between researchers and potential participants and transfer of prisoners out of recruitment prisons to other sites prior to scheduled interviews occurring.

Data from nine participants were excluded; six had lengthy delays in release dates (still in prison at time of initial data analysis), two revealed at post-release interviews they had not met pre-incarceration IDU eligibility criteria and one due to a technical error in data collection. The resultant sample of 400 participants included $108(27 \%)$ recruited from low-security, $111(28 \%)$ from medium-security and 181 (45\%) from high-security prisons. Participants' median sentence length was 206 days (IQR 109-381), and the median time between baseline interview and release from prison was 33 days (IQR 13-62 days).

\section{Demographics}

The median age of participants was 36 years (IQR 3042). Seventeen percent of participants identified as Aboriginal and/or Torres Strait Islander and 90\% were born in Australia. Most participants (83\%) had not completed high school, and approximately half (49\%) reported government payments as their primary source of income prior to incarceration. Twenty-eight percent of participants reported living in unstable accommodation in the 6 months prior to incarceration. A quarter of participants (25\%) reported being removed from parents' care as children. Almost two thirds (64\%) reported being a parent or primary caregiver to a child/ren, and of these, almost half $(46 \%)$ reported involvement of child protection and one in five $(20 \%)$ reported having ever had children removed from their care (Table 2).

Incarceration and Pre-Incarceration Offending History

The median number of reported prior adult incarcerations was five (IQR 3-9), and 179 (45\%) participants reported being incarcerated at least once as a juvenile. Most participants (85\%) reported being under the influence of drugs (drugs alone or in combination with alcohol) at the time of committing the offence(s) for which they were currently imprisoned. Almost half (40\%) reported their offending occurred because they needed money to purchase alcohol or other drugs (Table 2).

\section{Physical and Mental Health}

Infectious diseases were the most commonly reported general health condition (75\%); $74 \%$ self-reported a previous hepatitis $\mathrm{C}$ diagnosis, and confirmatory seroprevalence of hepatitis $\mathrm{C}$ antibodies was $82 \%$. Seven percent of participants self-reported a previous diagnosis of hepatitis 
Table 2 Socio-demographics and pre-incarceration offending characteristics at baseline

\begin{tabular}{ll}
\hline Variable & $\begin{array}{l}N=400 \\
n(\%)\end{array}$ \\
\hline
\end{tabular}

Socio-demographics

Median age (years)(IQR)

$36(30-42)$

Aboriginal or Aboriginal and Torres $66(17)$ Strait Islander

Australian born

English as primary language spoken 388 (97) at home

Highest education level completed

Year 7-9 ${ }^{1}$

$168(42)$

Year $10-11$

$162(41)$

36 (9)

Certificate/Diploma

154 (39)

36 (9)

9 (4)

Other sources ${ }^{4}$

Accommodation type before prison

Private rental (single or shared)

113 (28)

80 (20)

Family member's home

73 (18)

Public housing

57 (14)

Temporary accommodation ${ }^{5}$

$36(9)$

23 (6)

Owner occupied

$111(28)$

Self-reported accommodation as unstable before prison

$100(25)$

257 (64)

118 (46)

Children removed from your care ${ }^{6}$

Incarceration and pre-incarceration offending

Median self-reported adult incarceration episodes (IQR)

Incarcerated as a Juvenile

179 (45)

Under the influence of alcohol or other drugs at the time of committing this offence?

Yes, drugs only

Yes, drugs and alcohol

104 (26)

Yes, alcohol only

Current sentence related to purchase drugs or alcohol

\footnotetext{
${ }^{1}$ Includes 13 who did not complete any high school education

${ }^{2}$ Includes 10 who completed tertiary education

${ }^{3}$ Government payments include unemployment benefits, study benefits and pensions
}

${ }^{4}$ Other sources include money from family and friends, withdrawn savings and begging

${ }^{5}$ Temporary accommodation includes staying with friends, boarding houses and crisis accommodation

${ }^{6}$ Among those reporting to be parents or caregivers

$\mathrm{B}$, and less than $1 \%$ self-reported a diagnosis of HIV. Other commonly reported health conditions included dental (70\%), musculoskeletal (45\%) and respiratory (31\%) health conditions. One in five participants $(20 \%)$ reported having been diagnosed with an acquired brain injury. Most participants $(83 \%)$ reported a previous mental health diagnosis. Almost half (44\%) reported three or more mental health diagnoses. Depression (66\%), anxiety (50\%) and drug-induced psychosis (34\%) were the most common mental health diagnoses (Table 3).

Table 3 Health and mental health characteristics at baseline

$\begin{array}{ll}\text { Variable } & \begin{array}{l}N=400 \\ n(\%)\end{array} \\ \end{array}$

Self-reported physical health conditions

Chronic infectious disease 298 (75)

Ever tested positive for hepatitis C 293 (74)

DBS hepatitis C antibody prevalence 328 (82)

Dental condition $281(70)$

Musculoskeletal condition 180 (45)

Respiratory disease 122 (31)

Hearing or vision condition $100(25)$

Neurological disease $\quad 48$ (12)

Circulatory disease $\quad 46$ (12)

Metabolic disease 13 (3)

Self-reported mental health conditions

Any mental health condition $\quad 332$ (83)

Three or more mental health conditions 175 (44)

Depression $262(66)$

Anxiety disorder 201 (50)

Drug-induced psychosis 137 (34)

Post-traumatic stress disorder $\quad 85$ (21)

Bipolar disorder 66 (17)

Personality disorder ${ }^{1} \quad 63(16)$

Schizophrenia $56(14)$

Panic disorder 54 (14)

Other mental health disorder $^{2} \quad 47$ (12)

${ }^{1}$ Includes anti-social, borderline and other personality disorders

${ }^{2}$ Includes Schizoaffective disorder, attention deficit hyperactivity disorder, adjustment disorder, autism spectrum disorder, disorder, eating disorder, obsessive compulsive disorder and paedophilia 
Table 4 Illicit drug use characteristics at baseline

\begin{tabular}{lllll}
\hline Illicit substance* & Ever used & $\begin{array}{l}\text { Used month before } \\
\text { incarceration } \\
n(\%)\end{array}$ & $\begin{array}{l}\text { Ever injected } \\
n(\%)\end{array}$ & $\begin{array}{l}\text { Injected month } \\
\text { before incarceration } \\
(\%)\end{array}$ \\
\hline Cannabis & $374(94)$ & $258(65)$ & NA & NA \\
Crystal methamphetamine & $372(93)$ & $315(79)$ & $361(90)$ & $296(74)$ \\
Heroin & $355(89)$ & $226(57)$ & $344(86)$ & $220(55)$ \\
Speed & $355(89)$ & $75(19)$ & $132(33)$ & $72(18)$ \\
Ecstasy & $286(72)$ & $33(8)$ & $168(42)$ & $11(3)$ \\
Cocaine & $277(69)$ & $52(13)$ & $242(61)$ & $28(7)$ \\
Prescription opiates & $273(68)$ & $81(20)$ & $55(14)$ & $72(18)$ \\
Benzodiazepines & $259(65)$ & $122(31)$ & $176(44)$ & $9(2)$ \\
Buprenorphine (Subutex and & $225(56)$ & $49(12)$ & $26(7)$ & $31(8)$ \\
$\quad$ Suboxone) & $126(32)$ & $19(5)$ & $61(15)$ & $2(1)$ \\
Methadone & $122(31)$ & $18(5)$ & $13(3)$ & \\
Pharmaceutical stimulants & & &
\end{tabular}

*Illicit substances include pharmaceutical substances and medications not obtained via personal prescription

\section{Substance Use}

The median age of reported first episode of IDU was 17 years (IQR 15-20). The most commonly used substances by any route of administration and injected in the month prior to incarceration were crystal methamphetamine (79\% and $74 \%$, respectively), heroin $(57 \%$ and 55\%), benzodiazepines (31\% and $2 \%$ ) and prescription opioids (20\% and $18 \%$ ) (Table 4$)$.

Of the 324 participants who responded to questions about IDU in prison, ${ }^{2} 211$ (65\%) reported ever injecting in prison, $130(40 \%)$ reported injecting during their current sentence and $81(25 \%)$ reported injecting during a previous incarceration but not their current sentence. Among those reporting IDU during their current sentence, $60(46 \%)$ reported injecting in the past month, reporting a median of eight injections over this period (IQR 2-28). The drugs most commonly injected during the current sentence were crystal methamphetamine $(65 \%)$, buprenorphine-naloxone $(45 \%)$ and heroin $(35 \%)$.

\section{Alcohol and Other Drug Treatment Service Utilisation}

Seventy-two percent $(n=288)$ of participants reported lifetime use of OST. Half $(n=143 ; 50 \%)$ of those ever on OST reported receiving OST at some point during

\footnotetext{
$\overline{2}$ These were considered by participants to be sensitive questions and a number of participants declined to answer them.
}

their current sentence. Of the 121 who were receiving OST at the time of baseline interview, $95 \%$ were receiving methadone.

Most participants reported accessing some form of non-OST AOD treatment in the community $(69 \%)$ or prison $(75 \%)$ in their lifetime. The most commonly accessed community and prison-based non-OST AOD programs were detoxification services (45\%) and group therapy (65\%), respectively. Non-OST AOD treatment was accessed by $17 \%$ of participants in the 12 months before prison and $48 \%$ of participants during their current sentence. Over half of participants (54\%) expressed a desire to access non-OST AOD services upon release from prison (Table 5).

\section{Discussion}

Despite people with histories of incarceration and IDU typically exhibiting significant and complex health and social disadvantage $[16,19,33]$ and exceptionally high rates of reincarceration [22], little is known about their experiences after release from prison. Our lack of understanding of individual, social and service access factors associated with patterns of drug use, health and criminogenic outcomes after release from prison impedes the development of effective policies and practices. The PATH Cohort Study purposively recruited people in prison for post-release follow-up who reported a history of regular IDU in the months immediately prior 
Table 5 Non-opioid substitution therapy drug and alcohol treatment service utilisation at baseline

\begin{tabular}{|c|c|c|c|c|}
\hline $\begin{array}{l}\text { Non-OST treatment service type (multiple } \\
\text { responses allowed }\end{array}$ & $\begin{array}{l}\text { Ever accessed in } \\
\text { community } \\
N=398 \\
n(\%)\end{array}$ & $\begin{array}{l}\text { Ever accessed in } \\
\text { prison } \\
N=399 \\
n(\%)\end{array}$ & $\begin{array}{l}\text { Accessed during baseline } \\
\text { incarceration } \\
N=399 \\
n(\%)\end{array}$ & $\begin{array}{l}\text { Wanted to access } \\
\text { post-release } \\
N=393 \\
n(\%)\end{array}$ \\
\hline Any non-OST treatment & $273(69)$ & $301(75)$ & $192(48)$ & $211(54)$ \\
\hline Group therapy & $67(17)$ & $259(65)$ & $136(34)$ & $13(3)$ \\
\hline Individual counselling & $121(30)$ & $123(31)$ & $52(13)$ & $166(42)$ \\
\hline $\begin{array}{l}\text { Alcoholics or narcotics } \\
\text { anonymous }\end{array}$ & $79(20)$ & $85(21)$ & $37(9)$ & $28(7)$ \\
\hline Detox & $179(45)$ & NA & NA & $10(3)$ \\
\hline Residential rehabilitation & $121(30)$ & NA & NA & $20(5)$ \\
\hline One off information session & NA & $190(48)$ & $82(21)$ & NA \\
\hline Other service type* & $2(1)$ & $0(0)$ & $0(0)$ & $10(3)$ \\
\hline
\end{tabular}

*Other service type includes naltrexone implant, drug court and voluntary urinary drug screens

to incarceration and who were due for release within 12 weeks of interview. The PATH Cohort is unique in the Australian prisoner research context, where followup of prisoners has typically been much shorter $[15,34]$ or relied solely on secondary data linkage $[9,35]$. The PATH Cohort is also unique in that it focuses on people who were injecting drugs regularly at the time of incarceration. This cohort represents a particularly complex and vulnerable group at high risk of a range of negative post-release outcomes. The detailed data collected at pre-release baseline, which we present in this paper, will couple with post-release follow-up data to provide unprecedented insights into the trajectories of this population over coming years to help inform pre- and postrelease health and support programs.

The baseline characteristics of our cohort show expected indicators of socio-economic disadvantage, such as low educational attainment, accommodation instability and a reliance on government welfare and crime for income, and also an extensive history of incarceration that is reflected in community-recruited cohorts of PWID [31]. Our findings also show extensive patterns of intergenerational disadvantage. One quarter of respondents reported being removed from their parents as children, and almost half of participants who were parents or caregivers reported child protection involvement, and one in five reported having had their children removed from their care. While incarceration and intergenerational disadvantage has been extensively documented [36-38], the fact that almost two thirds of our cohort are the primary caregiver to a child underscores the substantial long-term social and economic costs associated with the nexus between disadvantage, dependent drug use and incarceration.

Substances reported as being injected by participants in the month prior to and during incarceration reflect recent trends in drug markets and drug-related harms in Australia and the emergence of methamphetamine as a commonly injected drug in Australia [2, 39]. In local Victorian drug trend surveillance, the proportion of PWID reporting methamphetamine as their most commonly injected drug increased from $17 \%$ in 2012 to $30 \%$ in 2016, although heroin remains the most commonly injected drug among survey respondents $(66 \%)$ [40]. The over-representation of methamphetamine use in our cohort reflects other Australian law enforcement data on drug use among police arrestees [41, 42] and findings from a recent Australian study reporting methamphetamine as the most commonly injected drug prior to incarceration among prisoners with a history of IDU in New South Wales [43].

Among those who answered the question, almost two thirds of our sample reported ever injecting in prison (across a median of five previous incarcerations) and $40 \%$ reported injecting during their current sentence. While this prevalence of in-prison IDU is higher than other Australian studies, it is not unexpected given we purposively recruited participants frequently injecting prior to incarceration (at least monthly in the 6 months prior to incarceration) compared to others who recruited prisoners reporting any injecting in the 3 months preincarceration [42] or a lifetime history of injecting [23, 34]. The prevalence of pre-incarceration and in-prison methamphetamine injecting in our cohort, and trends in 
methamphetamine use among Australian PWID more broadly, highlights the need for effective methamphetamine treatment responses. Analysis of PWID cohort study data in Melbourne showed an association between drug first injected and current injecting drug preferences, and highlighted the need for flexible harm reduction and drug treatment services that respond to changing patterns of drug use [31]. This need for services that respond to changing drug use trends should also apply to law enforcement responses and programs available in prison. While OST is widely available in Victorian prisons (and was the most commonly accessed prison drug treatment program in our cohort), prison programs for methamphetamine dependence have relied primarily on group and individual counselling. While counselling treatment approaches to methamphetamine dependence have demonstrated some success in community settings [44], their effectiveness in prison settings is yet to be established [45]. A forthcoming trial of pharmacotherapy for the management of methamphetamine dependence [46] may result in new drug treatments suited to implementation in prison.

Our experience recruiting cohort participants provides insights into non-random and targeted prison recruitment strategies. Targeting recruitment to recent and frequent IDU meant that screening for eligibility via prison administrative data was not possible. Instead, we relied mostly on researchers spending extensive time in prisons and building trust and rapport with prisoners. Our recruitment strategies initially relied upon promoting the study via posters and program workers, but were soon modified to focus more on researchers' direct engagement with prisoners in their units or at clinical or program visits. The ability to communicate the purpose of the study and answer questions about participation on the spot, and to encourage information about the study spreading via word-of-mouth, meant that direct engagement strategies were the most fruitful by far. In the context of aiming to recruit participants close to their expected release date, the most significant recruitment challenge was the transfer of eligible and interested prisoners out of recruitment prisons before scheduled interviews could take place. Movement between prison sites late in a sentence is also a challenge for the provision of effective transitional support for people leaving prison, particularly if transitional support service providers differ according to prison region or site.

Our study has limitations associated with sampling and reporting. First, as noted above, our eligibility criteria and limitations in administrative data meant that we were unable to implement a random sampling or consecutive sampling recruitment approach, and as such, findings may not be generalizable to the broader Victorian prison population with histories of IDU. More than twice the number of people recruited submitted an expression of interest form to participate in the study. While a small number were excluded from the study because they did not meet eligibility criteria (e.g. preincarceration drug use, release dates beyond the study recruitment period), inability to participate in the study mostly occurred because of transfers to other prisons prior to individuals becoming eligible to complete baseline surveys on the basis of expected release date. Movements between prisons overwhelmingly occur due to operational requirements rather than being based on prisoner behaviours or characteristics, and this inability to participate is therefore not expected to result in meaningful sampling bias. The restriction of the study to three recruitment sites and the variation in numbers recruited at each site may also introduce some recruitment bias. This paper is mostly based on self-reported responses and may be susceptible to reporting and recall bias, particularly for questions that refer to participants' pre-incarceration experiences. However, for most survey domains, future record linkage will provide objective data to validate self-report and other measures (e.g. lifetime IDU history) that could not be collected in any other way. While our sample includes a higher proportion of Indigenous participants (17\%) relative to the general Victorian prison population (7.8\%) [12], it is unclear the extent to which this represents an overrepresentation of prisoners with IDU histories. The sample also includes participants with a median sentence length of 206 days. In Victoria, $25.8 \%$ of prisoners have an effective sentence length of less than 1 year. The shorter sentence length in our sample may reflect the types of crimes committed by people incarcerated for drug-related offences. Shorter sentences in this group bear further exploration in future analysis given access to some prison health programs (e.g. hepatitis $\mathrm{C}$ treatment) is restricted to those on longer sentences or residing in non-remand prisons. Finally, incarcerated women with IDU histories were not recruited and the study will therefore not reflect the experiences and specific challenges faced by women after release from prison [47]. The original study design included an over-sample of 100 female prisoners; however, operational pressures at the women's prisons in Victoria precluded their 
recruitment (e.g. dealing with an influx of women prisoners and engaging in substantial new construction).

Our description of the baseline characteristics of PATH participants shows a cohort with substantial challenges with respect to physical and mental health, drug use, individual family histories, history of offending, educational attainment and employment history. Instability of accommodation and state involvement in the care of children further demonstrate the complexity of providing adequately coordinated, holistic care and support for this population during their reintegration into the community. Future analyses of prospective data will identify unmet community needs and describe the timevariant and time-invariant factors associated with specific health, drug use and criminogenic trajectories in this population. We aim to provide novel information to support policy and practice change related to the timing, targeting and modes of interventions designed to improve this population's health and social outcomes.

Acknowledgements This research was funded by a National Health and Medical Research Council Project Grant (APP1029915). MS, PD and SK are supported by NHMRC Senior Research Fellowships. We gratefully acknowledge the support provided to the Burnet Institute by the Victorian Government Operational Infrastructure Support Program. The authors would also like to acknowledge the participants involved in the PATH Study for the time and knowledge they contributed. Thanks to the Burnet Institute fieldwork team for their tireless efforts with data collection.

Open Access This article is distributed under the terms of the Creative Commons Attribution 4.0 International License (http:// creativecommons.org/licenses/by/4.0/), which permits unrestricted use, distribution, and reproduction in any medium, provided you give appropriate credit to the original author(s) and the source, provide a link to the Creative Commons license, and indicate if changes were made.

\section{References}

1. Carpentier C, Royuela L, Montanari L, Davis P. The global epidemiology of drug use in prison. In: Kinner SA, Rich JD, editors. Drug use in prisoners: epidemiology, implications, and policy responses. Oxford: Oxford University Press; 2017. p. 17-42.

2. Australian Institute of Health and Welfare. The health of Australia;s prisoners 2015. Canberra: ACT: AIHW; 2015.

3. Fazel S, Baillargeon J. The health of prisoners. Lancet. 2010;377:956-65.

4. Reekie JM, Levy MH, Richards AH, et al. Trends in prevalence of HIV infection, hepatitis B and hepatitis C among
Australian prisoners - 2004, 2007, 2010. MJA. 2014;200(5):277-80.

5. Brinkley-Rubinstein. Incarceration as a catalyst for worsening health. Health Justice. 2013;1(3).

6. Markowitz FE. Mental illness, crime, and violence: risk, context, and social control. Aggress Violent Behav. 2011;16(1):36-44.

7. Graffam J, Shinkfield AJ. The life conditions of Australian ex-prisoners: an analysis of intrapersonal, subsistence, and support conditions. Int $J$ Offender Ther Comp Criminol. 2012;56(6):897-916.

8. Hellard ME, Hocking JS, Crofts N. The prevalence and the risk behaviours associated with the transmission of hepatitis $\mathrm{C}$ virus in Australian correctional facilities. Epidemiol Infect. 2004;132:409-15.

9. Degenhardt L, Larney S, Kimber J, Gisev N, Farrell M, Dobbins T, et al. The impact of opioid substitution therapy on mortality post-release from prison: retrospective data linkage study. Addiction. 2014;109(8):1306-17.

10. Winter RJ, Stoové M, Degenhardt L, et al. Incidence and predictors of non-fatal drug overdose after release from prison among people who inject drugs in Queensland, Australia. Drug Alcohol Depend. 2015;153:43-9.

11. Australian Bureau of Statistics. 2016 Census QuickStats. 2018. http://www.quickstats.censusdata.abs.gov.au/census services/getproduct/census/2016/quickstat $/ \overline{2}$ ?opendocument. Accessed 13 Jan 2019.

12. Australian Bureau of Statistics. Prisoners in Australia [4517.0] 2018. 2018. http://www.abs.gov.au/ausstats/abs@. nsf/Lookup/by\%20Subject/4517.0 2018 Main\%20 Features $\sim$ Aboriginal $\% 20$ and $\% 20$ Torres $\% 20$ Strait $\% 20$ Islander\%20prisoner\%20characteristics\%20 13. Accessed 13 Jan 2019.

13. Australian Institute of Health and Welfare. Alcohol, tobacco \& other drugs in Australia. 2019. https://www.aihw.gov. $\mathrm{au} / \mathrm{reports/alcohol/alcohol-tobacco-other-drugs-}$ australia/contents/priority-populations/aboriginal-andtorres-strait-islander-people. 2019. Accessed 7 Feb 2019.

14. Kariminia A, Butler T, Levy M. Aboriginal and nonaboriginal health differentials in Australian prisoners. Aust New Z J Public Health. 2007;31(4):366-71.

15. Baldry E, McDonnell D, Maplestone P, Peeters M. Australian prisoners' post-release housing. Curr Issues Crim Just. 2003;15(2):155-69.

16. Binswanger IA, Nowels C, Corsi KF, et al. Return to drug use and overdose after release from prison: a qualitative study of risk and protective factors. Addict Sci Clin Pract. 2012;7(1):3-3.

17. Freudenberg N, Moseley J, Labriola M, Daniels J, Murrell C. Comparison of health and social characteristics of people leaving New York City jails by age, gender, and race/ethnicity: implications for public health interventions. Public Health Rep. 2007;122(6):733-743.

18. Hamilton M. People with complex needs and the criminal justice system. Curr Issues Crim Just. 2010;22(2):307-24.

19. Winter RJ, Young JT, Stoove M, Agius PA, Hellard ME, Kinner SA. Resumption of injecting drug use following release from prison in Australia. Drug Alcohol Depend. 2016;168:104-11.

20. Kirwan A, Quinn B, Winter R, Kinner SA, Dietze P, Stoove M. Correlates of property crime in a cohort of recently 
released prisoners with a history of injecting drug use. Harm Reduct J. 2015;12:23.

21. Department of Justice \& Regulation CV. Department of Justice \& Regulation Annual Report 2016-17. Melbourne, VIC: Victorian Government; 2017.

22. Larney S, Burns L. Evaluating health outcomes of criminal justice populations using record linkage: the importance of aliases. Eval Rev. 2011;35(2):118-28.

23. Kinner SA, Jenkinson R, Gouillou M, Milloy M. High-risk drug-use practices among a large sample of Australian prisoners. Drug Alcohol Depend. 2012;126(1-2):156-60.

24. Kinner SA. Continuity of health impairment and substance misuse among adult prisoners in Queensland, Australia. Int $J$ Prison Health. 2006;2(2):101-13.

25. Farrell M, Marsden J. Acute risk of drug-related death among newly released prisoners in England and Wales. Addiction. 2008;103(2):251-5.

26. Karaminia A, Butler TG, Corben SP, et al. Extreme causespecific mortality in a cohort of adult prisoners - 1988 to 2002: a data-linkage study. Int J Epidemiol. 2007;36(2): 310-6.

27. Carrotte E, Dietze P, Kirwan A. Syringe coverage and Australian NSPs. Centre for Research Excellence into Injecting Drug Use. Policy Brief 9. Melbourne: Burnet Institute; 2015.

28. ABS. Prisoners in Australia 2015, cat. no. 4517. 2016. http://www.abs.gov.au/ausstats/abs@.nsf/Lookup/by\%20 Subject/4517.0 2015 Main\%20Features Key\%20 findings $\sim 1$. Accessed 9 July 2016.

29. Corrections Victoria. Monthly prisoner and offender statistics 2014-15 to 2017-18. 2016; www.corrections.vic.gov. au/utility/publications+manuals+and+statistics/monthly+ prisoner+and+offender+statistics. Accessed 10 Dec 2018.

30. CreativityCorp. Mobile Data Studio: Versions 7 \& 8. Manjimup, WA: CreativityCorp. 2011.

31. Horyniak D, Higgs P, Jenkinson R, Degenhardt L, Stoové $\mathrm{M}$, Kerr T, et al. Establishing the Melbourne injecting drug user cohort study (MIX): rationale, methods, and baseline and twelve-month follow-up results. Harm Reduct J. 2013;10(11):11.

32. StataCorp. Stata Statistical Software: Release 14. College Station, TX: StataCorp LCC. 2015.

33. Binswanger IA, Nowels C, Corsi KF, et al. "From the prison door right to the sidewalk, everything went downhill": a qualitative study of the health experiences of recently released inmates. Int J Law Psychiatry. 2011;34(4):249-55.

34. Kinner SA, Lennox N, Williams GM, et al. Randomised controlled trial of a service brokerage intervention for exprisoners in Australia. Contemp Clin Trials. 2013;36(1): 198-206.

35. Larney S, Toson B, Burns L, Dolan K. Effect of prison-based opioid substitution treatment and post-release retention in treatment on risk of re-incarceration. Addiction. 2012;107(2):372-80.

36. Foster H, Hagan J. Incarceration and intergenerational social exclusion. Soc Probl. 2007;54(44):399-433.

37. van de Rakt M, Joseph M, Paul N. The long-term effects of paternal imprisonment on criminal trajectories of children. $J$ Res Crime Delinq. 2011;49(1):81-108.

38. Kinner SA, Borschmann R. Inequality and intergenerational transmission of complex adversity. Lancet Public Health. 2017;2(8):e342-3.

39. Stafford J, Breen C. Australian Drug Trends 2015. Findings from the Illicit Drug Reporting System (IDRS). Sydney: NSW: National Drug and Alcohol Research Centre, UNSW; 2016.

40. Aitken C, Lloyd B, Dietz P. Victorian Drug Trends 2016: findings from the Illicit Drug Reporting System (IDRS). Sydney: NSW: National Drug and alcohol Centre, UNSW; 2017.

41. Coghlan S, Gannoni A, Goldsmid S, Patterson E, Willis M. Drug use monitoring in Australia: 2013-14 report of drug use among police detainees. Canberra: Australian institute of Criminology; 2015.

42. Coghlan S, Goldsmid S. Findings from the DUMA program: methamphetamine drug market trends. Canberra: Australian Insititute of Criminology; 2015.

43. Cunningham EB, Hajarizadeh B, Amin J, Bretana N, Dore GJ, Degenhardt L, et al. Longitudinal injecting risk behaviours among people with a history of injecting drug use in an Australian prison setting: the HITS-p study. Int J Drug Policy. 2018;54:18-25.

44. Ciketic S, Hayatbakhsh R, McKetin R, Doran CM, Najman JM. Cost-effectiveness of counselling as a treatment option for methamphetamine dependence. J Subst Abus. 2015;20(4):239-46.

45. de Andrade D, Ritchie J, Rowlands M, Mann E, Hides L. Substance use and recidivism outcomes for prison-based drug and alcohol interventions: a systematic review. Epidemiol Rev. 2018;40(1):121-33.

46. McKetin R, Dean OM, Baker AL, Carter G, Turner A, Kelly $\mathrm{PJ}$, et al. A potential role for $\mathrm{N}$-acetylcysteine in the management of methamphetamine dependence. Drug Alcohol Rev. 2017;36(2):153-9.

47. Baldry E. Women in transition: from prison to.... Curr Issues Crim Just. 2010;22(2):253-67.

48. Andrews DA, Bonta JL. Level of Service InventoryRevised: Screening Version (LSI-R-SV) [User's manual]. Toronto: Multi-Health Systems. 1998.

Publisher's Note Springer Nature remains neutral with regard to jurisdictional claims in published maps and institutional affiliations. 